\title{
Distributed antenna-coupled transition edge sensors
}

Peter K. Day, Henry G. LeDuc, Richard A. M. Lee, C. Darren Dowell, Jonas Zmuidzinas

Peter K. Day, Henry G. LeDuc, Richard A. M. Lee, C. Darren Dowell, Jonas Zmuidzinas, "Distributed antenna-coupled transition edge sensors," Proc. SPIE 6275, Millimeter and Submillimeter Detectors and Instrumentation for Astronomy III, 62751R (27 June 2006); doi: 10.1117/12.672690

SPIE Event: SPIE Astronomical Telescopes + Instrumentation, 2006, Orlando, Florida, United States 


\title{
Distributed Antenna-Coupled Transition Edge Sensors
}

\author{
Peter K. Day ${ }^{a}$, Henry G. LeDuc ${ }^{a}$, Richard A. M. Lee ${ }^{b}$, C. Darren Dowell ${ }^{a}$ and Jonas \\ Zmuidzinas $^{b}$ \\ ${ }^{a}$ Jet Propulsion Laboratory, California Institute of Technology, Pasadena, CA 91109; \\ ${ }^{b}$ California Institute of Technology, Pasadena, CA 91125
}

\begin{abstract}
We describe progress toward realizing a new architecture for focal plane arrays for the Submillimeter and FarInfrared (FIR) bands. This architecture is based on a detector design utilizing distributed hot-electron transition edge sensors (TES) coupled to slot antenna elements. Arrays utilizing this type of detector can be considerably easier to manufacture than membrane-isolated TES arrays, because the need for micro-machining is eliminated. We present background and rationale for this new array architecture and details of a new antenna design for an imaging polarimeter, which yields greater bandwidth than past designs. In addition, we describe a cryogenic facility for testing these arrays.
\end{abstract}

Keywords: bolometer, TES, detector array, slot antenna

\section{INTRODUCTION}

The next generation of astronomical instruments for Submillimeter and FIR wavelengths will require arrays of tens of thousands of pixels. For example, the proposed Caltech-Cornell Atacama Telescope will need 32,000 pixels at 620, 450, 350 and 200 microns to fully sample its field of view. The Single Aperature Far-Infrared Observatory (SAFIR) mission, still a priority of the science community, will require an array with approximately $128 \times 128$ detectors with a very high sensitivity of around $10^{-18} \mathrm{~W} / \mathrm{Hz}^{1 / 2}$ to be photon noise limited.

When they are fielded, the SCUBA-2 arrays will represent the state-of-the-art in this wavelength range, incorporating thousands of pixels at 850 and 350 microns, ${ }^{1}$ which is an order of magnitude more than current pioneering instruments such as the Submillimeter High Angular Resolution Camera II (SHARC-II) ${ }^{2,3}$ and Bolocam. ${ }^{4}$ Micromachined silicon nitride arrays of TES bolometers along the lines of those used for SCUBA-II could be extended by yet another order of magnitude in pixel count, but would likely be expensive.

A pixel architecture that is both easier to manufacture and compatible with the high sensitivity needed for a space mission would be desirable. In the area of millimeter-wave detectors, detector designs incorporating antenna-coupled TES bolometers have been proposed and demonstrated. ${ }^{5,6}$ In these devices, power from a planar antenna is transmitted down a transmission line to a load resistor, which is thermally isolated on a small island along with a TES. Antenna-coupled pixels have several important advantages. First, only the load resistor and TES need to be thermally isolated, so the micromachined structures are much simpler. Second, the specific heat of the absorber is avoided, so the detectors can be much faster. Third, no backshort is needed as the antenna lies on a substrate with high dielectric constant, so most of the power is radiated into the substrate (hence, most of the power incident on the antenna through the substrate can be efficiently absorbed). The millimeter-wave antenna-coupled devices have additional benefits: phased array antennas can be used that have narrow beam widths that couple easily to a telescope, and filters can be inserted in the microstrip transmission line between antenna and TES to define the pass-band.

For very short wavelengths, microstrip cannot be used to transport power between antenna and detector because because the microstrip becomes lossy at frequencies higher than the gap frequency, about 700 Ghz for niobium. Proposed antenna-coupled FIR/Submillimeter detectors therefore collocate the sensor with the antenna. ${ }^{7,8}$ In these cases, the power collected by the antenna is dissipated directly in the TES and the thermal

Further author information: (Send correspondence to P.K.D.)

P.K.D: E-mail: Peter.K.Day@jpl.nasa.gov, Telephone: 6265775582

Millimeter and Submillimeter Detectors and Instrumentation for Astronomy III, edited by Jonas Zmuidzinas, Wayne S. Holland, Stafford Withington, William D. Duncan, Proc. of SPIE Vol. 6275, 62751R, (2006) · 0277-786X/06/\$15 - doi: 10.1117/12.672690

Proc. of SPIE Vol. 6275 62751R-1 
(a)

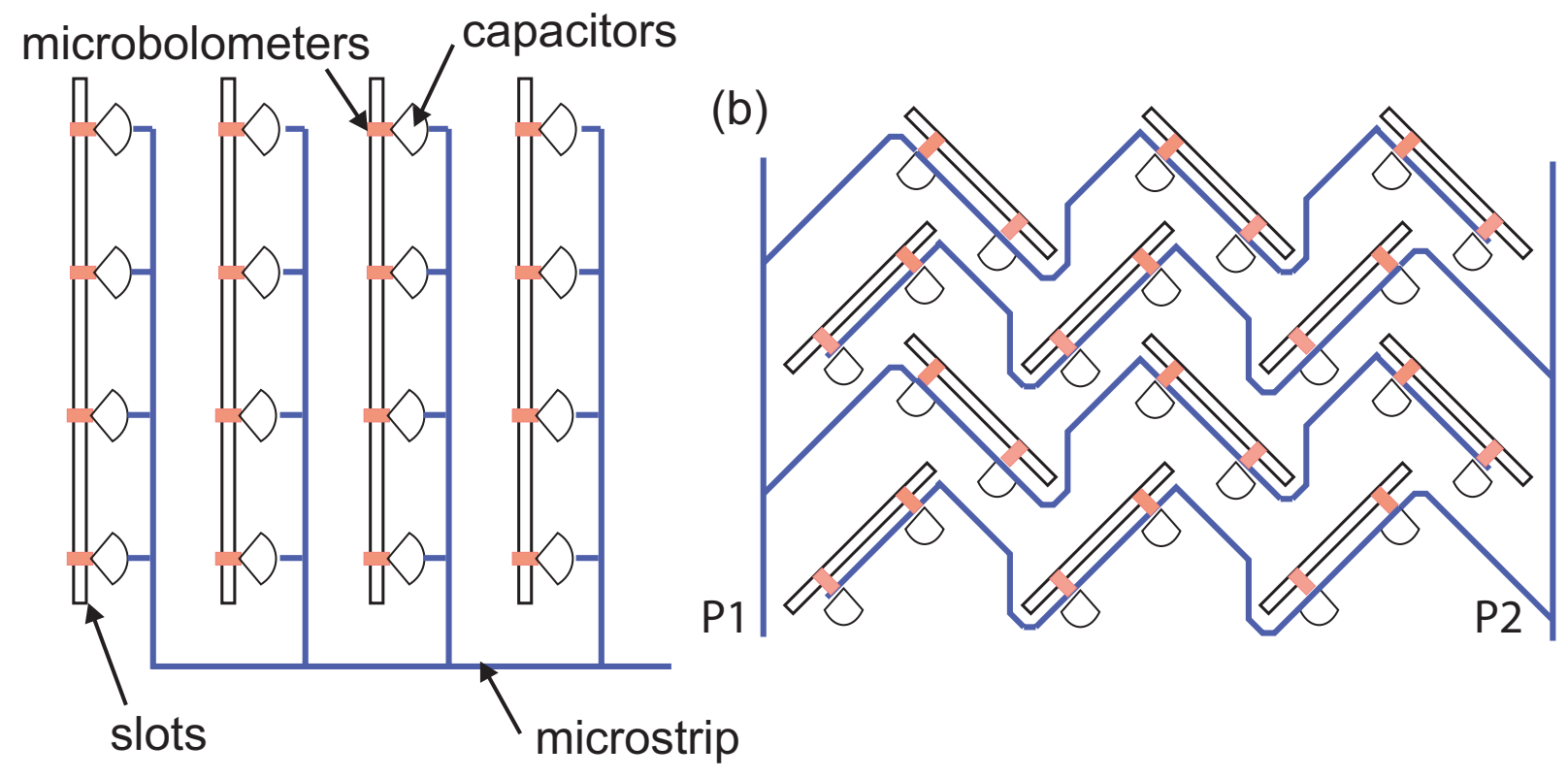

Figure 1. (a) Design of the (a) single polarization and (b) dual polarization pixels. An array of slots on a ground-plane is tapped directly by an array of microbolometers. Typically arrays of $8 \times 8$ or 16x16 microbolometers will be used, depending on the desired pixel size. A reduced number is shown for clarity.

isolation is achieved through the thermal decoupling of the electrons and phonons in the TES, ie. the "hot electron" effect. The thermal conductance is then proportional to the volume of the sensor, prescribing a very small TES volume for low enough $G$. One drawback of the simple hot-electron antenna-coupled detector is that the active area of the antenna is much smaller than the pixel size, which is $k f \lambda$ for a given $f /$ number. For Nyquist sampling, $k=0.5$. Hence, to efficiently fill the focal plane, an aperture-defining dielectric lens must be placed in front of each antenna.

\section{PIXEL DESIGN}

Our new pixel design ${ }^{9}$ makes use of an array of slot antennas, which is essentially a scaled down version of the microstrip coupled slot array antennas used at millimeter wavelengths. Rather than feeding the slots with microstrips, we directly tap the slot array with an array of TES microbolometers (fig. 1). The microbolometers are made from a thin film of a superconductor with an appropriate $T_{c}$ with dimensions in the range of one to tens of microns on a side. The normal resistance of the microbolometer is chosen to match to the antenna. A microbolometer is placed at each of the feedpoints where, in the millimeter-wave version of the antenna, a microstrip line would tap the antenna. To allow for biasing of the microbolometers, a DC connection is made between the microbolometer and the ground plane of the slot on one side of the slot. On the other side, the microbolometer is connected through a capacitor large enough to present a short at the frequency of the antenna.

The microbolometers for a particular pixel are all biased in parallel with a bias line that snakes between the slots. The bias line is implemented in microstrip even though it only carries the low frequency output of the bolometer. Use of microstrip in this case conveniently hides the wiring behind the antenna groundplane. The arrangement of slots in fig. 1(b) allows for polarization sensitive imaging as separate bias lines may be used for the different slot directions. For non-polarization sensitive applications, a single bias line may be used.

The parallel bias scheme effectively impedance matches the antenna array and the SQUID amplifier that is used to for the readout of the detector. Each of the microbolometers has a normal state resistance on the order of $30 \Omega$ in order to match to the antenna impedance. The impedance seen by the SQUID is the parallel combination of $\sim 64-256$ microbolometers, depending on the pixel size (see below). The resulting normal state resistance of 


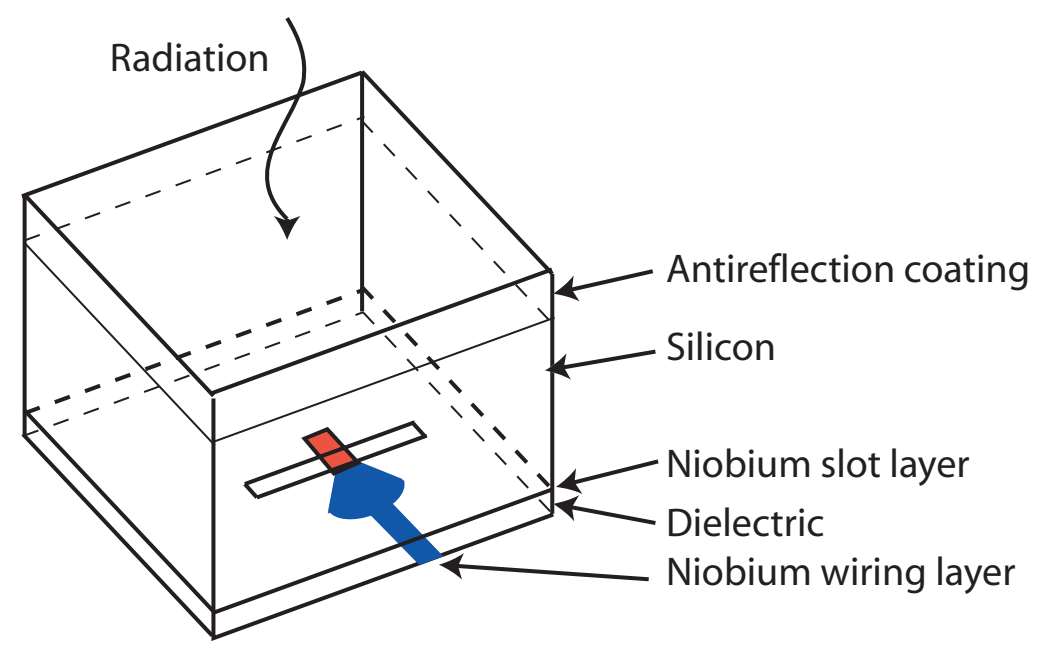

Figure 2. The antennas are illuminated though the back of the silicon wafer on which they are fabricated. Because the substrate has a high dielectric constant, the antenna efficiently absorbs radiation in this direction. An antireflection coating matches to free space or a resonant $(\lambda / 4)$ substrate may be used.

the parallel combination, on the order of a few hundred milliohms, allows the use of a bias point in the middle of the transition curve, where low noise operation is typically observed, while maintaining compatibility with current SQUID multiplexers.

As with the millimeter-wave antennas, the slot array pixels are intended to fill a focal plane without the need for lenses or feedhorns. In contrast with the microstrip coupled devices, the detectors we describe here are multimode detectors. Because the radiation collected by the slot array is combined incoherently these antennas do not have a narrow beam pattern, but rather absorb radiation over a broad range of angles similar to a resistive absorber. These detector arrays are therefore comparable to the arrays of bare absorbers used for SHARC-II ${ }^{2,3}$ and SCUBA-II. ${ }^{1}$

Just as with the single-moded microstrip-coupled antennas, the slot spacing, $\delta_{s}$, should satisfy $\delta_{s}<\lambda / \sqrt{\epsilon_{r}}$, where $\epsilon_{r}$ is the relative dielectric constant of the substrate, to avoid coupling to substrate modes ${ }^{6}$. For the single mode devices, coupling to substrate modes reduces the efficiency of the detector. In the case of the multimode detectors, the incoming wave drives the slots coherently, so satisfying the criterion on $\delta_{s}$ will prevent the scattered

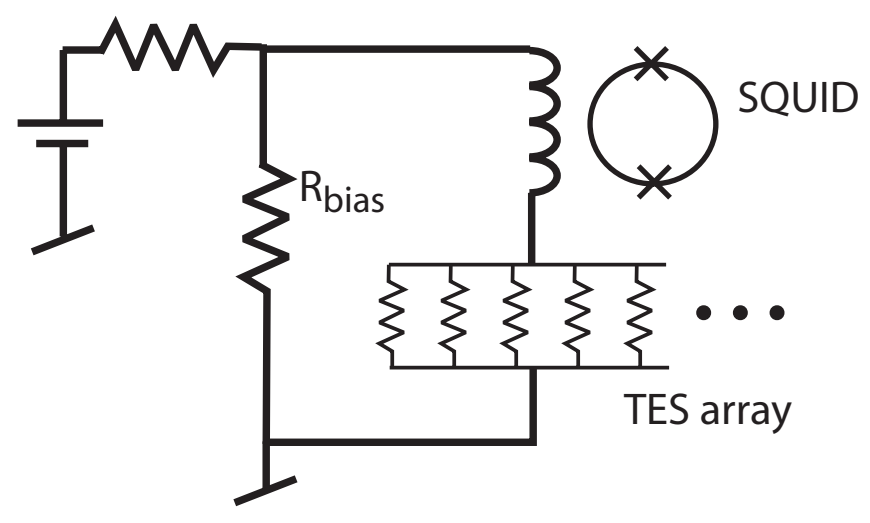

Figure 3. The array of microbolometers corresponding to a particular pixel are biased in parallel. This scheme effectively matches the antenna impedance of $\sim 30 \Omega$ to the SQUID multiplexer, which requires a TES resistance of less than an ohm. 
(a)

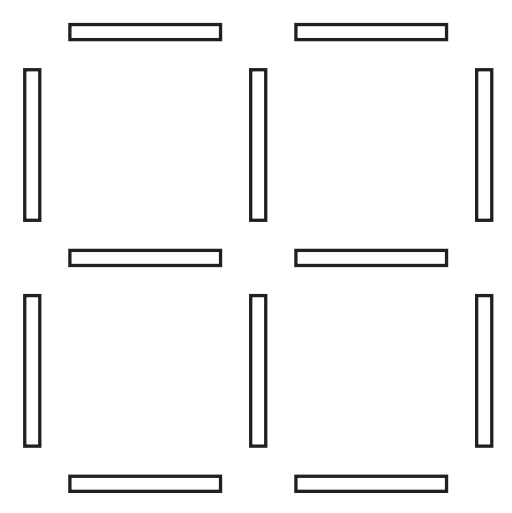

(b)

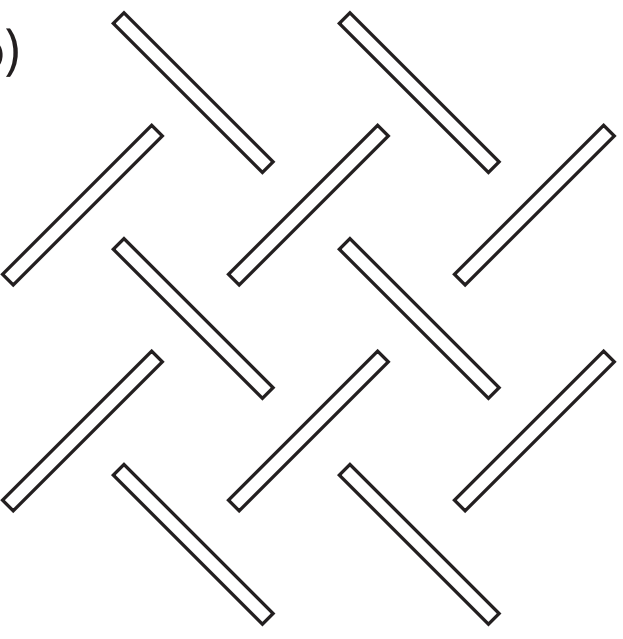

Figure 4. (a) Geometry of the 'L' antenna. (b) The new 'H' geometry allows the slots to be longer relative the spacing between them, improving the bandwidth

wave from the antenna from coupling into substrate modes, which would be a source of cross-talk. For $f / 4$ and a silicon substrate $\left(\epsilon_{r}=11.5\right)$, the number of microbolometers is approximately 64 for $0.5 f \lambda$ pixels (Nyquist sampling) and 256 for $f \lambda$ pixels.

The thermal noise is

$$
\mathrm{NEP}_{\text {thermal }}=\sqrt{4 k_{B} T^{2} G}
$$

where $G$ is the combined thermal conductance between electrons and phonons for all of the microbolometers in a pixel and is proportional to the total volume. Hence, the NEP of an individual microbolometer would be $\sqrt{N}$ times smaller than the combination if it were biased separately.

\section{ANTENNA DESIGN}

The antennas described here are essentially scaled down versions of the millimeter-wave microstrip-coupled slot arrays. ${ }^{6,10}$ The incoherently combined FIR detectors will have broad beam patterns relative to the coherently combined slot arrays, but the impedance of the antennas will be similar. For example, if we illuminate the incoherent slot arrays with the same beam generated by the coherent antenna $(\sim \mathrm{f} / 4.5)$, by reciprocity the voltage across the individual slots must be identical in either case. Hence we can use the same method to calculate the antenna impedance.

Here we describe a new wider-bandwidth version of the dual polarization slot array antenna that can be used for both FIR and millimeter wave focal planes. The old and new geometries, which we will refer to as the ' $\mathrm{L}$ ' and ' $\mathrm{H}$ ' geometries, respectively, are depicted in fig. 4. The ' $\mathrm{H}$ ' geometry can be generated from the ' $\mathrm{L}$ ' geometry by rotating all of the slots by 45 degrees and lengthening them. The main difference with the new design is that the slots can be made longer relative to the lattice spacing while maintaining the same gap between slots.

The impedance of the antennas is calculated using the same method-of-moments procedure used for microstripcoupled slot antennas ${ }^{11,12}$ and slot arrays. ${ }^{10}$ In this method, the electric field in the slots is expanded in terms of a set of appropriate basis functions,

$$
E_{\alpha}(x, y)=\sum_{i} V_{\alpha, i} f_{\alpha, i}(x, y)
$$

where $\alpha=\{x, y\}$ and we make the approximation that $E_{x}=0$ for $\hat{x}$-directed slots and $E_{y}=0$ for $\hat{y}$-directed slots. The admittance kernal

$$
Q_{\alpha \beta}\left(k_{x}, k_{y}\right)=\frac{1}{Z_{0}}\left[\left(\frac{\epsilon_{1} k}{\gamma_{1}}+\frac{\epsilon_{2} k}{\gamma_{2}}\right) \delta_{\alpha \beta}-\left(\frac{1}{k \gamma_{1}}+\frac{1}{k \gamma_{2}}\right) p_{\alpha} p_{\beta}\right]
$$




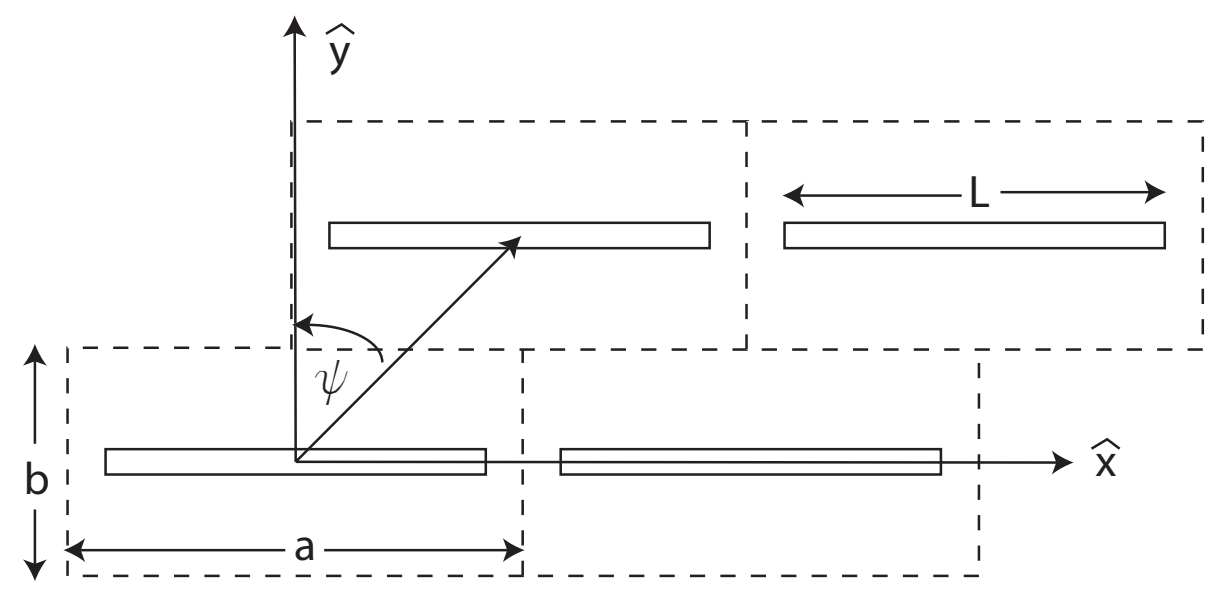

Figure 5. Skewed lattice corresponding to the infinite array case of the new dual-polarization antenna. Only one slot direction is shown.

where $\delta_{\alpha \beta}$ is the Kronecker delta function, $\alpha, \beta=x$ or $y, \vec{p}=\left(k_{y}, k_{x}, 0\right), \epsilon_{1,2}$ are the dielectric constants above and below the antenna plane, $\gamma_{i}^{2}=\epsilon_{i} k^{2}-k_{x}^{2}-k_{y}^{2}\left(\operatorname{Im}\left\{\gamma_{1}\right\} \leq 0, \operatorname{Im}\left\{\gamma_{2}\right\} \geq 0\right)$, and $Z_{0}$ is the impedance of free space.

Using the Galerkin method, a matrix equation is written for the unknown basis function amplitudes in terms of the currents in the slots:

$$
I_{i}=\sum_{j} Y_{i j} V_{j}
$$

where $\vec{I}$ is the vector of overlap integrals between the slot currents and the basis functions and

$$
Y_{i j}=\frac{-1}{4 \pi^{2}} \int_{-\infty}^{\infty} \int_{-\infty}^{\infty} d k_{x} d k_{y} Q_{\alpha \beta}\left(k_{x}, k_{y}\right) \widetilde{f}_{i}^{*}\left(k_{x}, k_{y}\right) \widetilde{f}_{j}\left(k_{x}, k_{y}\right)
$$

where $\tilde{f}_{i}$ are the fourier transforms of the basis functions, and the proper form of $Q_{\alpha \beta}$ is used depending on whether basis functions $i$ and $j$ belong to parallel or perpendicularly oriented slots. Once the basis function amplitudes are evaluated, the impedance at the feed points is determined from the ratio of the voltage to the current at those points.

In the case of an infinite array of slots, only wave vectors that reflect the periodicity of the lattice are allowed and the integrals are replaced by sums. For the ' $\mathrm{H}$ ' antenna, it is convenient to use a skewed lattice and slots oriented in the $\hat{x}$ direction (fig. 5 ). In this case, the wave vectors satisfy ${ }^{13}$

$$
\begin{aligned}
k_{x, m n} & =\frac{2 m \pi}{a} \\
k_{y, m n} & =\frac{2 n \pi}{b}-\frac{2 m \pi}{b} \tan \psi,
\end{aligned}
$$

where $\psi=\pi / 4$. The admittance matrix becomes

$$
Y_{i j}=\frac{-1}{a b} \sum_{m n} Q_{\alpha \beta}\left(k_{x, m n}, k_{y, m n}\right) \tilde{f}_{i}^{*}\left(k_{x, m n}, k_{y, m n}\right) \tilde{f}_{j}\left(k_{x, m n}, k_{y, m n}\right)
$$

where $m, n$ are integers.

The method-of-moments calculations were performed using 11 piecewise sinusoidal basis functions per slot. ${ }^{11}$ Increasing the number of basis functions produced essentially the same results. We have calculated both the 

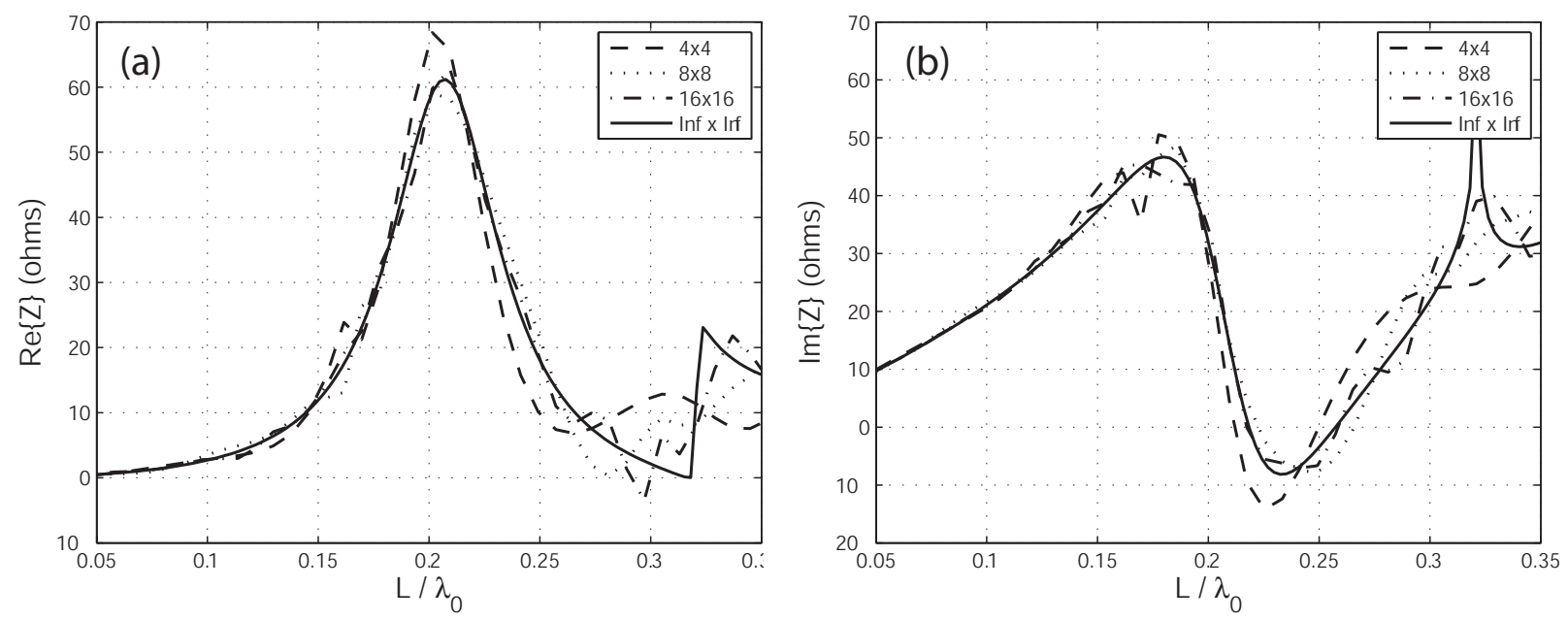

Figure 6. (a) Real and (b) imaginary parts of the impedance of the ' $\mathrm{H}$ ' antenna versus $L / \lambda_{0}$, where $L$ is the length of the slot and $\lambda_{0}$ is the free space wavelength, for $4 \times 4,8 \times 8,16 \times 16$ elements and infinite arrays. The lattice spacing is $1.3 L$, $W=0.04$ and the feed points are $\pm 0.38 \mathrm{~L}$.

infinite array and finite arrays of various numbers of elements up to 16 by 16 . As shown in fig. 6 , the impedance of the 16 by 16 array is very close to that of the infinite array.

We operate the antenna near the peak due to the first resonance in order to stay well below the frequency where grating lobes become an issue. For a feed point centered in the slot, the impedance at the peak is $\sim 150 \Omega$. Operating with such a large microbolometer resistance would be possible, but would lead to a parallel resistance that is still somewhat high for the SQUID readout. (For the millimeter wave version of the antenna, it would be difficult to make the impedance of the microstrip line this large.) In order to reduce the impedance of the antenna
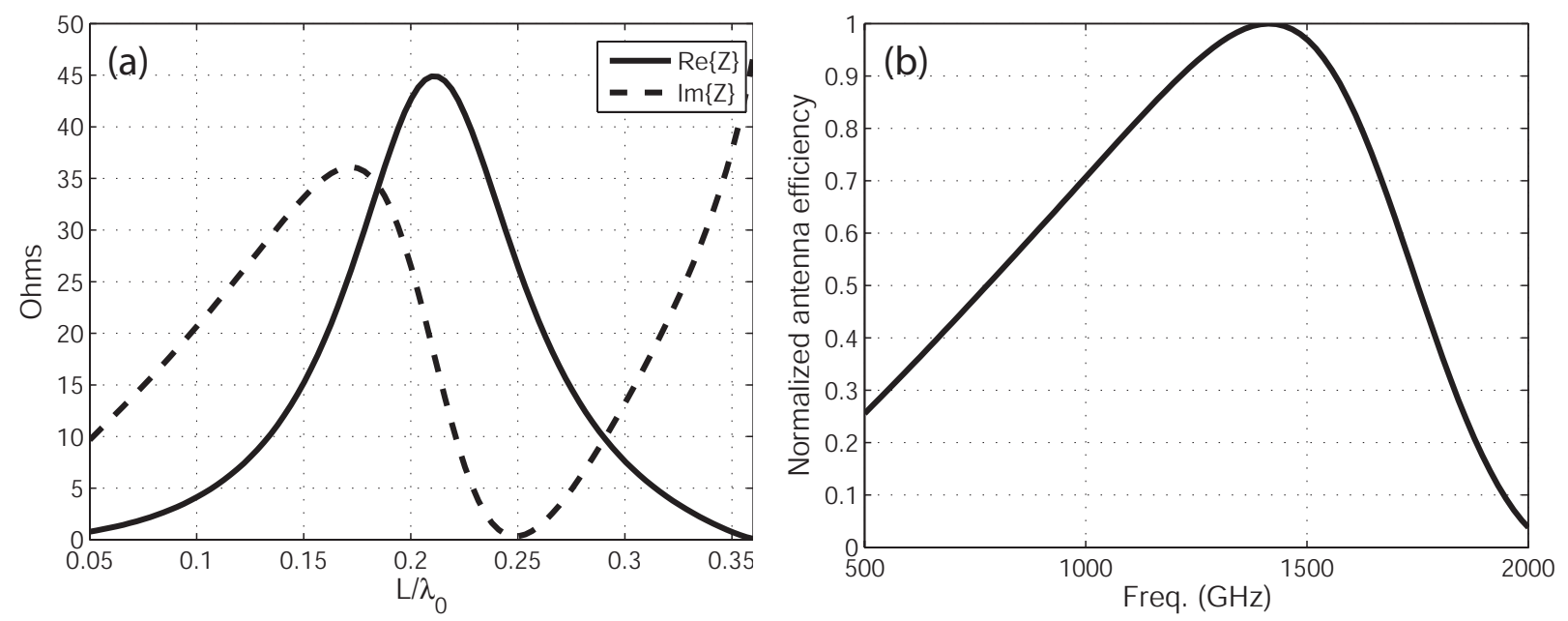

Figure 7. (a) Impedance of the dual polarization antenna. The substrate is silicon $(\epsilon=11.5)$ and the width of the slot is $0.04 \mathrm{~L}$. The feed points are located at $\pm 0.38 \mathrm{~L}$, and the lattice spacing is $1.1 \mathrm{~L}$. (b) Normalized efficiency of the antenna with $28 \Omega$ microbolometers. The length of the slot is chosen to center the pass band at $1.4 \mathrm{THz}$. The The bandwidth is large enough to cover the 1.3 and $1.5 \mathrm{THz}$ atmospheric windows with $90 \%$ efficiency. 


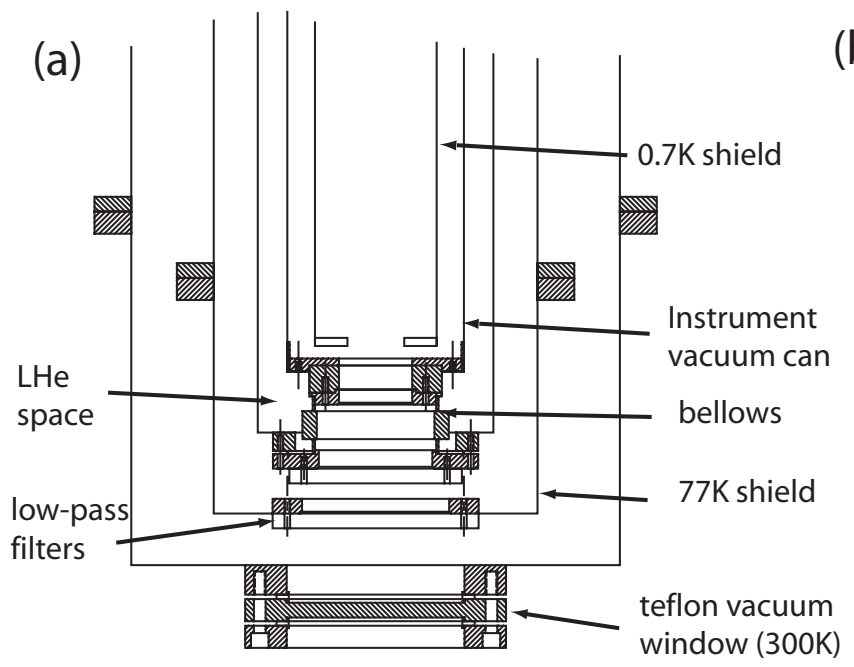

(b)

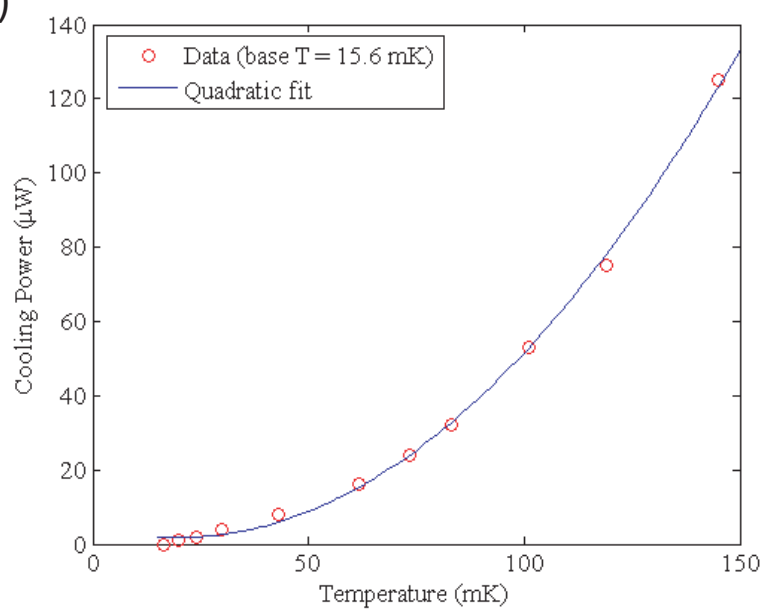

Figure 8. (a) Filtering scheme for the test cryostat. Radiation is admitted into the dewar through a teflon vacuum window. 300 and $250 \mathrm{~cm}^{-1}$ low pass filters are mounted on the $77 \mathrm{~K}$ radiation shield. A further band-specific low pass filter is mounted at $4 \mathrm{~K}$ and a bandpass filter is mounted on the $0.7 \mathrm{~K}$ (still) shield. (b) Temperature versus power loading curve for the dilution refrigerator. The in-band power expected to be absorbed by the mixing chamber with the filters configured for the $53 \mu \mathrm{m}$ band (highest loading) is approximately $25 \mu \mathrm{W}$, which would give an operating temperature of $70 \mathrm{mK}$.

we move the feed points toward the end of the slots. We use two feeds per slot in order to maintain symmetry (fig. 1(b)) which results in very low cross polarization. In the case of the infinite array, the cross-polarization coupling vanishes. For finite arrays, coupling between nearest neighbor slots of differing polarizations vanishes, so the only cross-polarization coupling can be from more distant neighbors around the edges of the array where the symmetry is broken.

Feeding the slots near the ends makes the antenna more inductive, so that the imaginary part of the impedance no longer goes to zero at the peak in the real part, as is the case for a center-fed slot. A purely real impedance is obtained by operating on the high frequency side of the peak (fig. 7), which results in a somewhat asymmetric pass band and a bandwidth $\delta f(3 d B) / f_{\text {center }}=0.77$ (for a lattice constant of $1.1 L$ ).

\section{TEST APPARATUS}

We are constructing a cryogenic facility for optical testing of detectors in the submm/FIR bands at temperatures of $100 \mathrm{mK}$ or even lower. The facility is based on an Oxford dilution refrigerator, to which we have added a dewar with an optical access port, purchased from Precision Cryogenics. The cryostat can receive radiation from a FIR fourier transform spectrometer (FTS) located in a separate vacuum chamber.

In order to get radiation into a standard top-loading dilution refrigerator, without the use of cryogenic vacuum windows, we are using a bellows to connect the dilution refrigerator's vacuum can to the vacuum jacket of the dewar (see fig. 8a). Because the instrument vacuum and the dewar vacuum are connected in this arrangement, we cannot use exchange gas to cool the sample. To obtain a fast cooldown time, we use a circulator capillary which makes a loop from outside the dewar through the helium bath to the mixing chamber and back. By blowing helium gas through the circulator, we can effectively pre-cool the refrigerator.

Before reaching the detector under test, the radiation passes through a reconfigurable set of filters that can be used to define bands at 220,130, and 58 microns. Two low pass filters are mounted on the $77 \mathrm{~K}$ shield which are common to all of the bands. A further low pass filter is mounted at $4 \mathrm{~K}$ and a band pass filter is mounted on the $0.7 \mathrm{~K}$ shield, which is connected to the still of the dilution refrigerator. The in-band radiative power from $300 \mathrm{~K}$ transmitted through the final filter is approximately $250 \mu \mathrm{W}$ for the 58 micron band configuration, which 


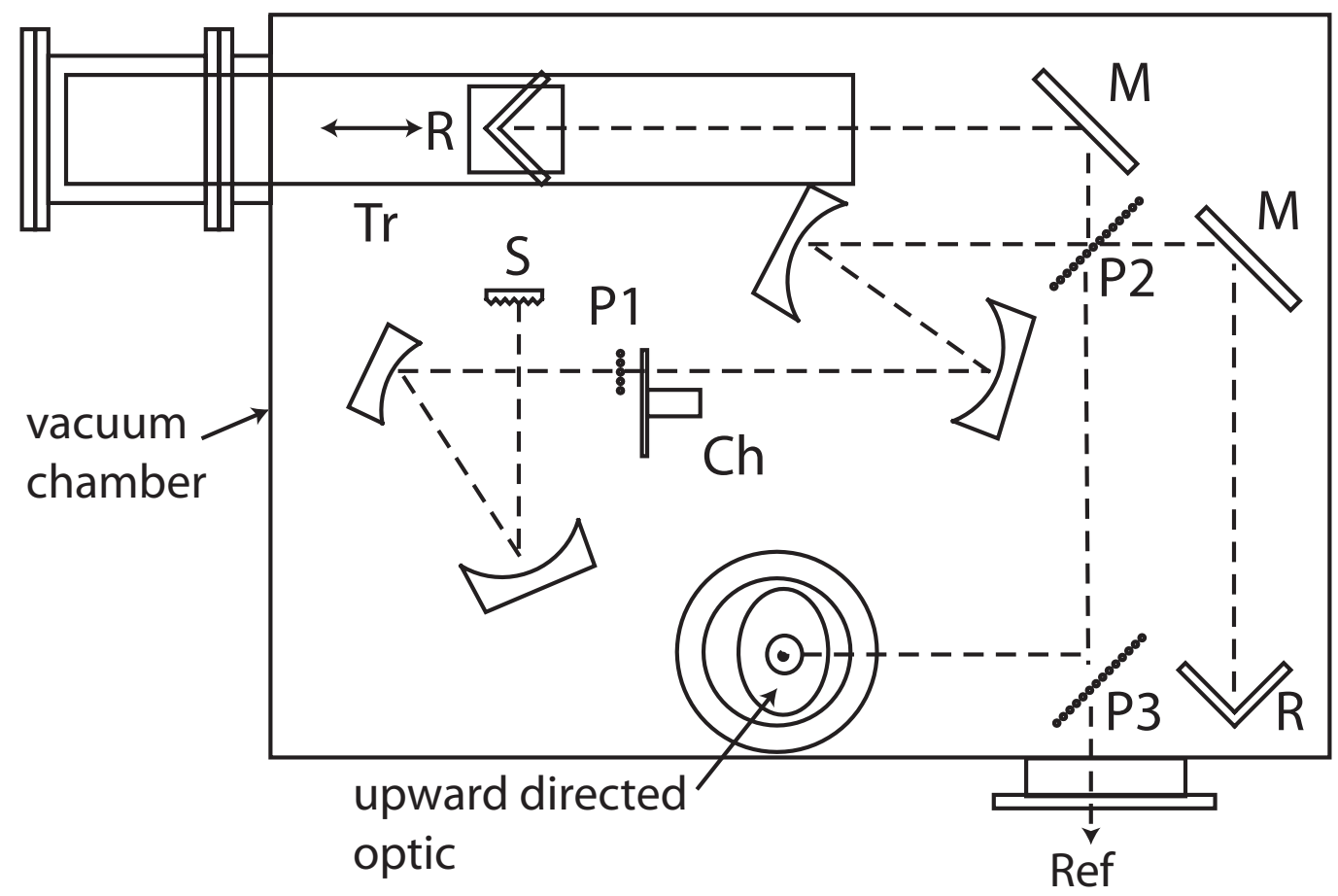

Figure 9. Layout of the far-Infrared Martin-Puplett polarizing spectrometer. The spectrometer resides in a turbopumped vacuum chamber. The source $(\mathrm{S})$ can be heated to $1000 \mathrm{~K}$. After passing through polarizer P1, the beam is chopped with a mechanical chopper (Ch). Polarizer (P2) functions as a broad-band beam splitter. The length of one arm of the interferometer is varied by moving a rooftop reflector $(\mathrm{R})$ on a translation stage (Tr). The maximum path difference is approximately $1 \mathrm{~m}$. A third polarizer (P3) splits the beam between two output ports. An upward-directed optic couples the beam into the dilution refrigerator above. A reference bolometer system can measure the beam through a side-directed port.

is the highest loading case. The cooling power curve for the dilution refrigerator is shown in fig. 8(b). Assuming an emissivity of 0.1 for the surface of the mixing chamber gives an estimated base temperature of $70 \mathrm{mK}$ with the window open.

The fourier transform spectrometer (fig. 9) is a Martin-Puplett interferometer, which uses a polarizing grid as the beam splitter. ${ }^{14}$ The radiation source is a ceramic disk that can be heated to $1000 \mathrm{~K}$. The spectrometer resides inside a vacuum chamber that can be connected to the dewar of the dilution refrigerator through an evacuated tube.

\section{CONCLUSIONS}

We have described a new pixel design that is applicable to large focal plane arrays for submillimeter and FIR wavelengths. An improved version of the dual polarization slot array antenna has been designed that increases the bandwidth over an earlier design. ${ }^{9}$ Arrays of dual-polarization antenna-coupled detectors bring several practical benefits to astronomical instruments in the field. Maximum sensitivity is achieved over the detector field of view, which is important for many imaging applications. Common mode signals such as atmospheric emission and thermal drift of the focal plane are rejected optimally in the measurement of polarization since each diffraction beam is observed in both polarizations and since the distributed TES arrays should be electrically well matched for each polarization. The monolithic approach significantly reduces complexity as compared to separate arrays observing orthogonal polarizations, ortho-mode transducers, or stacked polarization-sensitive detectors. 


\section{REFERENCES}

1. W. Duncan et al. SCUBA-2: Application of LTD technology. AIP Conference Proceedings, LTD-9, 605:577$580,2002$.

2. G. M. Voellmer, C. A. Allen, M. J. Amato, S. R. Babu, A. E. Bartels, D. J. Benford, R. J. Derro, C. D. Dowell, D. A. Harper, M. D. Jhabvala, S. H. Moseley, T. Rennick, P. J. Shirron, and J. G. Staguhn. Design and fabrication of two-dimensional semiconducting bolometer arrays for the high-resolution airborne wideband camera (HAWC) and the submillimeter high angular resolution camera II (SHARC-II). Proc. SPIE, 4855:63$72,2002$.

3. C. D. Dowell, C. A. Allen, S. R. Babu, M. M. Freund, M. B. Gardner, J. Groseth, M. D. Jhabvala, A. Kovacs, D. C. Lis, S. H. Moseley, T. G. Phillips, Silverberg R. F., G. M. Voellmer, and H. Yoshida. Sharc-II: A caltech submillimeter observatory facility camera with 384 pixels. Proc. SPIE, 4855:73-87, 2002.

4. J. Glenn, J. J. Bock, G. Chattopadhyay, S. F. Edgington, A. E. Lange, J. Zmuidzinas, P. D. Mauskopf, B. Rownd, L. Yuen, and P. A. R. Ade. Bolocam: a millimeter-wave bolometric camera. Proc. SPIE, 3354:326-334, May 2002.

5. C. L. Hunt, J. J. Bock, P. K. Day, A. Goldin, A. E. Lange, H. LeDuc, A. Vayonakis, and J. Zmuidzinas. Transition-edge superconducting antenna-coupled bolometer. Proc. SPIE, 3354:318-321, May 2002.

6. A. Goldin, J. J. Bock, C. Hunt, A. E. Lange, H. LeDuc, A. Vayonakis, and J. Zmuidzinas. Design of broadband filters and antennas for SAMBA. Proc. SPIE, 3354:163-171, May 2002.

7. M. Nahum and J. M. Martinis. Ultasensitive-hot-electron microbolometer. Appl. Phys. Lett., 63(22):30753077, Nov 1993.

8. B. S. Karasik, W. R. McGrath, M. E. Gershenson, and A. V. Sergeev. Photon-noise-limited direct detector based on disorder-controlled electron heating. J. Appl. Phys., 87(10):7586-7588, May 2000.

9. P. K. Day, H. LeDuc, A. Goldin, C.D. Dowell, and J. Zmuidzinas. Proc. SPIE, 5498:857-865, 2002.

10. A. Goldin, J. J. Bock, A. E. Lange, H. LeDuc, A. Vayonakis, and J. Zmuidzinas. Antennas for bolometric focal plane. Nuclear Instruments and Methods in Physics Research Section A, 520:390-392, 2004.

11. M. Kominami, D. Pozar, and D. Schaubert. Dipole and slot elements and arrays on semi-infinite substrates. AP, 33(6):600-607, 1985.

12. J. Zmuidzinas and H. G. LeDuc. Quasi-optical slot antenna SIS mixers. IEEE Trans. Microwave Theory Tech., MTT-40:1797-1804, 1992.

13. Craig Scott. The Spectral Domain Method in Electromagnetics. Artech House, Inc.: Norwood, MA, 1998.

14. D. H. Martin and E. Puplett. Polarized interferometric spectrometry for the millimeter and submillimeter spectrum. Infrared Phys., 10:105-109, 1969. 